\title{
Allogeneic cardiosphere-derived cells (CAP-1002) in critically ill COVID-19 patients: compassionate-use case series
}

\author{
Siddharth Singh ${ }^{1} \cdot$ Tarun Chakravarty $^{1} \cdot$ Peter Chen $^{2} \cdot$ Akbarshakh Akhmerov $^{1} \oplus$ - Jeremy Falk ${ }^{2}$. Oren Friedman ${ }^{1,2}$. \\ Tanzira Zaman ${ }^{2} \cdot$ Joseph E. Ebinger ${ }^{1} \cdot$ Mitch Gheorghiu $^{1} \cdot$ Linda Marbán $^{3} \cdot$ Eduardo Marbán $^{1} \cdot$ Raj R. Makkar ${ }^{1}$
}

Received: 28 April 2020 / Accepted: 5 May 2020 / Published online: 12 May 2020

(c) The Author(s) 2020

\begin{abstract}
There are no definitive therapies for patients with severe acute respiratory syndrome coronavirus-2 (SARS-CoV-2) infection. Therefore, new therapeutic strategies are needed to improve clinical outcomes, particularly in patients with severe disease. This case series explores the safety and effectiveness of intravenous allogeneic cardiosphere-derived cells (CDCs), formulated as CAP-1002, in critically ill patients with confirmed coronavirus disease 2019 (COVID-19). Adverse reactions to CAP-1002, clinical status on the World Health Organization (WHO) ordinal scale, and changes in pro-inflammatory biomarkers and leukocyte counts were analyzed. All patients $(n=6$; age range 19-75 years, 1 female) required ventilatory support (invasive mechanical ventilation, $n=5$ ) with $\mathrm{PaO}_{2} / \mathrm{FiO}_{2}$ ranging from 69 to 198 . No adverse events related to CAP-1002 administration were observed. Four patients (67\%) were weaned from respiratory support and discharged from the hospital. One patient remains mechanically ventilated as of April 28th, 2020; all survive. A contemporaneous control group of critically ill COVID-19 patients $(n=34)$ at our institution showed $18 \%$ overall mortality at a similar stage of hospitalization. Ferritin was elevated in all patients at baseline (range of all patients $605.43-2991.52 \mathrm{ng} / \mathrm{ml}$ ) and decreased in 5/6 patients (range of all patients $252.89-1029.90 \mathrm{ng} / \mathrm{ml}$ ). Absolute lymphocyte counts were low in $5 / 6$ patients at baseline (range $0.26-0.82 \times 10^{3} /$ $\mu \mathrm{l}$ ) but had increased in three of these five patients at last follow-up (range $0.23-1.02 \times 10^{3} / \mu \mathrm{l}$ ). In this series of six critically ill COVID-19 patients, intravenous infusion of CAP-1002 was well tolerated and associated with resolution of critical illness in 4 patients. This series demonstrates the apparent safety of CAP-1002 in COVID-19. While this initial experience is promising, efficacy will need to be further assessed in a randomized controlled trial.
\end{abstract}

Keywords Coronavirus · Hyperinflammation · Cytokine storm · Cell therapy

\section{Introduction}

On April 28, 2020, the number of confirmed patients with SARS CoV-2 infection (COVID-19) reached $\sim 3$ million worldwide, with $>200,000$ deaths [45]. Although most cases are mild to moderate in severity, about $15 \%$ develop severe pneumonia, and nearly $5 \%$ progress to acute respiratory

Raj R. Makkar

Raj.Makkar@cshs.org

1 Cedars-Sinai Medical Center, Smidt Heart Institute, $127 \mathrm{~S}$. San Vicente Boulevard, Advanced Health Sciences Pavilion, Third Floor, Suite A3100, Los Angeles, CA 90048, USA

2 Division of Pulmonary and Critical Care Medicine, Department of Medicine, Cedars-Sinai Medical Center, Los Angeles, CA 90048, USA

3 Capricor Inc, Beverly Hills, CA 90211, USA distress syndrome and multiple organ failure [25, 49]. This worsening is predominantly driven by cytokine upregulation and an exaggerated yet maladaptive inflammatory response [35, 40, 47]. Direct viral infection and cytopathic effects may also play a role. In the sickest patients, a hyperimmune response characterized by cytokine storm leads to critical illness and end-organ dysfunction, with high mortality. Mortality rates increase with severity of illness, and rates $>50 \%$ have been described in critically ill patients [7, 18, 48]. No measures, other than supportive therapy, have been found to be effective in treating COVID-19, constituting a major unmet medical need.

Immunomodulatory therapies under active investigation for COVID-19 include corticosteroids, anti-cytokine therapies (including monoclonal antibodies), convalescent plasma, intravenous immune globulin (IVIG) therapy, and inhibitors of specific inflammatory pathways, including 
therapy (including hydroxychloroquine and tocilizumab) per clinical practice protocols then in place for COVID-19 at CSMC. Lack of clinical improvement or deterioration despite standard care was the primary reason to evaluate patients for emergent administration of allogeneic CDCs. Exclusion criteria included known hypersensitivity to dimethyl sulfoxide (DMSO; a component of CAP-1002), prior stem cell therapy, pre-existing terminal illness (e.g., metastatic cancer), need for mechanical circulatory support and dialysis. In general, patients with multi-organ failure who were deemed to be too sick for any intervention were excluded from the study.

\section{Contemporaneous control group}

Given the compassionate use nature of the case series, there was no randomization. Nevertheless, we sought some basis for comparison of outcomes and clinical characteristics between the 6 CAP-1002-treated subjects reported here, and other critically ill patients simultaneously hospitalized for COVID-19 at our institution, with similar baseline characteristics. We, therefore, retrospectively characterized a set of patients admitted to CSMC on or after 3/1/2020 with RT-PCR confirmed SARS-CoV-2 infection, who required mechanical ventilation. To render the comparison as fair as possible, we quantified clinical status at 30.7 days of hospitalization (to match the average in the CAP-1002-treated subjects) and limited the analysis to patients who received anti-IL6 or anti-IL6 receptor agents during the hospitalization as standard-of-care therapy (as did all of the CAP1002-treated subjects). Patients were excluded if they: (1) did not have at least 30.7 days of follow-up from admission to the terminal event (death or hospital discharge); (2) were enrolled in any clinical trial requiring informed consent; and (3) had a tracheostomy placed prior to the current admission.

\section{Cell manufacturing}

CAP-1002 was manufactured by Capricor using reported methods $[11,41]$. Based on the IV infusion protocol and dose of CAP-1002 used in the HOPE-2 clinical trial of Duchenne muscular dystrophy (NCT03406780), we selected a dose of 150 million allogeneic CDCs to administer in this study. Cell product frozen concentrate was supplied in a volume of $10 \mathrm{ml}$ of cryogenic cell preservation solution consisting of $50 \%(\mathrm{v} / \mathrm{v})$ CryoStor ${ }^{\circledR}$ CS 10 containing 10\% DMSO, 40\% (v/v) HypoThermosol ${ }^{\circledR}$, and 10\% (v/v) Albumin (Human, HSA) 25\%. The primary container was a Daikyo Crystal Zenith ${ }^{\circledR}$ vial with chlorobutyl stopper that was stored at less than or equal to $-140{ }^{\circ} \mathrm{C}$ until thawed for use. Once thawed, each vial of CAP-1002 was diluted with $40 \mathrm{ml}$ of 5\% HSA for IV use only, containing the albumin component of human blood in a $60-\mathrm{ml}$ syringe.

\section{Cell administration}

CAP-1002 was administered IV via a peripheral access device (or a central venous access device, if it was available at the time of administration). Following administration of the cells, the patients were monitored for signs of clinical and biochemical improvement (or deterioration). If the patients continued to require significant supplemental oxygen after one week, they were re-evaluated for a second dose of cell administration as a booster dose. Prior to administration of the second dose, patients were premedicated with an antihistamine to mitigate any potential allergic reactions (which, though clinically insignificant, were noted in two patients in the HOPE- 2 clinical trial when premedication was omitted before the second dose). The rationale for a second administration of CAP-1002 is based on preclinical evidence demonstrating additional benefit with repeat dosing of CDCs. Importantly, a repeat administration of CDCs was not associated with sensitization or adverse immunologic responses in rodents $[2,36]$.

The final ready-to-use cell product in a total volume of $50 \mathrm{ml}$ was infused with a commercially available syringe pump [510(k) cleared for human use] set at an initial rate of $1 \mathrm{ml} / \mathrm{min}$ for $10 \mathrm{~min}( \pm 30 \mathrm{~s})$. If no signs or symptoms of a potential adverse reaction were observed, the infusion was continued at a rate of $4 \mathrm{ml} / \mathrm{min}$ for a total infusion time of $20 \mathrm{~min}( \pm 2 \mathrm{~min})$. Upon completion of infusion of the first vial of CAP-1002, and prior to infusion of the second vial, residual material was washed out of the IV tubing with a bolus of $5 \%$ HSA (approximately $4 \mathrm{ml}$ over $2 \min \pm 30 \mathrm{~s}$ ). The next cell dose of 75 million cells in the second $60-\mathrm{ml}$ syringe was administered to the subject at a rate of $4 \mathrm{ml} /$ $\mathrm{min}$, for a total infusion time of 12.5 ( $\pm 1 \mathrm{~min})$. After the second infusion, residual material was washed out of the IV tubing with a bolus of 5\% HSA (approximately $4 \mathrm{ml}$ over $2 \min \pm 30 s)$.

\section{RT-PCR}

RT-PCR was performed on nasopharyngeal specimens collected from patients who met Centers for Disease Control and Prevention clinical and/or epidemiological criteria for COVID-19 testing [10]. If RNA from SARS-CoV-2 was detected, the test was positive and the patient was considered infected with virus and presumed to be contagious. Laboratory test results were considered in the context of clinical observations and epidemiologic data in making a final diagnosis and patient management decisions. The test was developed and its performance characteristics were determined by CSMC Department of Pathology and Laboratory Medicine. The laboratory is certified under the Clinical Laboratory Improvement Amendments (CLIA) as qualified to perform high-complexity clinical laboratory testing. This 
test was validated, but independent review by FDA of this validation is pending.

\section{Statistical analysis}

Pooled data are presented as means \pm standard deviation (SD), range, and median. Due to small sample size, statistical tests comparing CAP-1002 and control groups were not performed.

\section{Results}

Six patients (age range 19-75 years, one woman) underwent IV infusion of CAP-1002 containing 150 million allogeneic CDCs. Consistent with the Berlin Criteria, all patients had acute respiratory distress syndrome (ARDS) prior to CAP-1002 infusion, with decreased $\mathrm{PaO}_{2} / \mathrm{FiO}_{2}$ ratios (range 69.0-198.0; median 142.5), diffuse bilateral pulmonary infiltrates on chest imaging $(n=6,100 \%)$, and evidence of preserved cardiac function on transthoracic echocardiography (LVEF range 50-75\%; median 55\%), suggesting a non-cardiac origin of respiratory failure [4]. Sequential organ failure and assessment (SOFA) scores ranged from 2 to 8 prior to cell administration [19]. All six patients had received an anti-IL6 agent, tocilizumab, prior to the CAP-1002 infusion. In addition to tocilizumab, patient 1 received lopinavir/ritonavir and the remaining patients received hydroxychloroquine for 5 days prior to the cell infusion. Patient 2 was 19 years old, but was morbidly obese (BMI 42.2). Patients 1, 3, 4, 5 had significant cardiovascular comorbidities (Table 1). All patients were intubated $(n=5)$ or required high-flow nasal cannula (HFNC) support $(n=1)$ at the time of enrollment. The one patient receiving HFNC support was deemed high risk for imminent intubation. The remaining intubated patients had been on invasive mechanical ventilation for 2-11 days prior to cell administration. The first patient was intubated at the time of enrollment, but extubated by the time of cell administration. This patient was determined to be at a high risk for reintubation given high oxygen requirements on HFNC and, therefore, underwent cell administration.

Blood cell counts, inflammatory markers and cytokine levels are summarized in Table 2 and Fig. 2. All patients had elevated CRP (Fig. 2a), ferritin (Fig. 2b), IL-6 (Fig. 2c) and $\mathrm{TNF} \alpha$ prior to cell infusion. Five patients had lymphopenia prior to infusion (Fig. $2 d$ ). IL $1 \alpha$ and IL-1 $\beta$ levels were not elevated in this cohort. Ferritin and CRP levels decreased in 5 out of 6 patients following cell infusion (Fig. 2b and a, respectively). IL-6 levels were increased in all six patients at baseline and decreased in four patients (Fig. 2c). IL-10 levels remained below reference range in one patient, decreased in three patients, and increased in two patients (Table 2).
Upon admission, two patients had mildly elevated cardiac troponin I levels (range of all patients $<0.02-0.07 \mathrm{ng} / \mathrm{ml}$; median $0.01 \mathrm{ng} / \mathrm{ml}$ ). During the course of the hospitalization, cardiac troponin I levels increased in 4 patients (range of all patients $<0.02-1.26 \mathrm{ng} / \mathrm{ml}$; median $0.13 \mathrm{ng} / \mathrm{ml}$ ) within 4-16 days of admission, but subsequently decreased in all these patients (range of all patients $<0.02-0.15 \mathrm{ng}$ / $\mathrm{ml}$; median $0.07 \mathrm{ng} / \mathrm{ml}$ ). Similarly, D-dimer levels were mildly elevated in four patients upon admission (range of all patients $0.34-2.22 \mu \mathrm{g} / \mathrm{ml}$; median $0.83 \mu \mathrm{g} / \mathrm{ml}$ ), increased in five patients within 4-17 days of admission (range of all patients $5.36-20.00 \mu \mathrm{g} / \mathrm{ml}$; median $20.00 \mu \mathrm{g} / \mathrm{ml}$ ), and subsequently decreased in four patients (range of all patients $1.53-20.00 \mu \mathrm{g} / \mathrm{ml}$; median $2.45 \mu \mathrm{g} / \mathrm{ml})$.

The clinical course of individual patients is summarized in Fig. 3. No patient experienced any complications related to CAP-1002 administration. Patients 1 and 4 received a second dose of CAP-1002, after pretreatment with diphenhydramine, likewise with no complications. All intubated patients improved clinically after cell infusion, except patients 4 and 6, who are still in ICU but remain stable. Patient 1 was already extubated at the time of infusion, and CAP-1002 was administered due to rising oxygen requirements (on HFNC) and concern for reintubation. Similarly, patient 6 received the infusion in the setting of respiratory deterioration and imminent risk for intubation. As of April 28, 2020 ( 5 days post-infusion), patient 6 has not been intubated. Patients 2, 3 and 5 were extubated on post-infusion days 3, 4 and 1, respectively. Except for patients 4 and 6, all patients were transferred out of the ICU and discharged from the hospital (Table 1). There were no overt instances of acute stroke, acute myocardial infarction, or acute pulmonary embolism during the hospitalization at the time of last follow-up. One patient (patient \#4) received additional antibiotics for a possible bacterial superinfection, although the diagnosis remains presumptive and no source has been identified. All patients are alive as of April 28, 2020, with a mean follow-up of $13.5 \pm 4.6$ days. This is in contrast to 6 deaths (18\%) noted among 34 similar patients who received treatment for COVID-19 in our medical ICU around the same time (notably all patients were treated with anti-IL6 therapy; Table 3).

\section{Discussion}

Administration of CAP-1002 as a compassionate therapy for patients with severe COVID-19 and significant comorbidities was safe, well tolerated without serious adverse events, and associated with clinical improvement, as evidenced by extubation (or prevention of intubation). All the critically ill patients who received CAP-1002 survived, and four out of six have been discharged. This is in contrast to high mortality 
Table 1 Clinical characteristics of critically ill patients with COVID-19

\begin{tabular}{|c|c|c|c|c|c|c|}
\hline Variable & Patient 1 & Patient 2 & Patient 3 & Patient 4 & Patient 5 & Patient 6 \\
\hline Age (years) & 75 & 19 & 55 & 71 & 60 & 58 \\
\hline Gender & Male & Male & Male & Female & Male & Male \\
\hline Weight $(\mathrm{kg})$ & 75.3 & 133.4 & 104.3 & 58.9 & 82.4 & 62.6 \\
\hline $\mathrm{BMI}\left(\mathrm{kg} / \mathrm{m}^{2}\right)$ & 23.2 & 42.2 & 33.6 & 26.4 & 32.2 & 22.3 \\
\hline Smoking & No & No & No & No & No & No \\
\hline Prior comorbidities & $\begin{array}{l}\text { Afib, HTN, HLD, } \\
\text { T2DM }\end{array}$ & Obesity & $\begin{array}{l}\text { HTN, HLD, } \\
\text { HFpEF, T2DM, } \\
\text { Obesity }\end{array}$ & $\begin{array}{l}\text { T2DM, HLD, } \\
\text { osteoporosis }\end{array}$ & $\begin{array}{l}\text { CKD, HTN, } \\
\text { obesity }\end{array}$ & None \\
\hline $\begin{array}{l}\text { Clinical presenta- } \\
\text { tion }\end{array}$ & $\begin{array}{l}\text { Fever, chills, } \\
\text { myalgia, cough, } \\
\text { dyspnea, diarrhea }\end{array}$ & $\begin{array}{l}\text { Fevers, chills, } \\
\quad \text { diarrhea, cough }\end{array}$ & $\begin{array}{l}\text { Malaise, ADHF } \\
\text { (edema, weight } \\
\text { gain) }\end{array}$ & $\begin{array}{l}\text { Fever, chills, SOB, } \\
\text { cough, emesis }\end{array}$ & $\begin{array}{l}\text { Fever, cough, } \\
\text { dyspnea, diar- } \\
\text { rhea, emesis }\end{array}$ & $\begin{array}{l}\text { Fevers, cough, } \\
\text { SOB, diarrhea }\end{array}$ \\
\hline $\begin{array}{l}\text { Time from symp- } \\
\text { tom onset to } \\
\text { admission (days) }\end{array}$ & 7 & 4 & 7 & 7 & 8 & 9 \\
\hline $\begin{array}{l}\text { Days hospitalized } \\
\text { (days) }\end{array}$ & 26 & 16 & 32 & 28 (ongoing) & 14 & 8 (ongoing) \\
\hline $\begin{array}{l}\text { Time from admis- } \\
\text { sion to critical } \\
\text { care transfer } \\
\text { (days) }\end{array}$ & 3 & 3 & 9 & 2 & 0 & 1 \\
\hline $\begin{array}{l}\text { Days from ICU } \\
\text { admission to first } \\
\text { infusion }\end{array}$ & 12 & 5 & 7 & 8 & 3 & 2 \\
\hline $\begin{array}{l}\text { Duration of MV } \\
\text { before CDC } \\
\text { (days) }\end{array}$ & $11^{\mathrm{a}}$ & 5 & 6 & 8 & 2 & $-^{\mathrm{b}}$ \\
\hline $\begin{array}{l}\text { Days from infusion } \\
\text { to extubation }\end{array}$ & $--^{\mathrm{a}}$ & 3 & 4 & $\begin{array}{l}\text { Patient is still } \\
\text { intubated, as of } \\
04 / 28 / 2020\end{array}$ & 1 & $-^{\mathrm{b}}$ \\
\hline $\begin{array}{l}\text { Total MV duration } \\
\text { (days) }\end{array}$ & 11 & 8 & 10 & Ongoing & 3 & $-^{\mathrm{b}}$ \\
\hline $\begin{array}{r}\mathrm{Pa} / \mathrm{FiO}_{2} \text { prior to } \\
\text { infusion }(\mathrm{P} / \mathrm{F})\end{array}$ & P/F: 69 & P/F: 145 & P/F: 173 & P/F: 140 & P/F: 198 & P/F: 93 \\
\hline $\begin{array}{l}\text { SOFA prior to } \\
\text { infusion }\end{array}$ & 3 & 4 & 5 & 8 & 5 & 2 \\
\hline Prior treatments & $\begin{array}{l}\text { Lopinavir-ritonavir } \\
(\times 5 \text { days }) \\
\text { Tocilizumab }(\times 1 \\
\text { dose })\end{array}$ & $\begin{array}{l}\text { HCQ }(\times 5 \text { days }) \\
\text { Tocilizumab }(\times 1 \\
\text { dose })\end{array}$ & $\begin{array}{l}\mathrm{HCQ}(\times 5 \text { days }) \\
\text { Tocilizumab }(\times 1 \\
\text { dose })\end{array}$ & $\begin{array}{l}\text { HCQ }(\times 5 \text { days }) \\
\text { Tocilizumab }(\times 1 \\
\text { dose })\end{array}$ & $\begin{array}{l}\mathrm{HCQ}(\times 5 \text { days }) \\
\text { Tocilizumab }(\times 1 \\
\text { dose })\end{array}$ & $\begin{array}{l}\mathrm{HCQ}(\times 5 \text { days }) \\
\text { Tocilizumab }(\times 1 \\
\text { dose })\end{array}$ \\
\hline Status & Discharged, alive & Discharged, alive & Discharged, alive & ICU, alive & Discharged, alive & ICU, alive \\
\hline
\end{tabular}

$A D H F$ acute decompensated heart failure, $A R D S$ acute respiratory distress syndrome, $C K D$ chronic kidney disease, $H C Q$ hydroxychloroquine, $H L D$ hyperlipidemia, $H T N$ hypertension, $H F p E F$ heart failure with preserved ejection fraction, $I C U$ intensive care unit, $M V$ mechanical ventilation, $S O B$ shortness of breath, T2DM Type 2 diabetes mellitus

${ }^{a}$ Patient received first dose shortly after extubation due to rising oxygen requirements and imminent reintubation

${ }^{b}$ Patient required high-flow nasal cannula support and received CAP-1002 to prevent invasive mechanical ventilation

rates $(\sim 50 \%)$ reported for critically ill patients with COVID19 [5]. Within our institution, an age- and gender-matched retrospectively assembled cohort of COVID-19 patients also showed higher mortality ( 6 of 34 patients) compared to the compassionate-use series ( 0 of 6 ), but statistical comparisons were not attempted given the small number of CAP1002-treated patients. Most patients receiving CAP-1002 also showed improvements in inflammatory markers, though to varying degrees. Similar to other COVID-19 cohorts, our patients exhibited elevated cardiac troponin I and D-dimer levels [37, 44]. These biomarkers, however, decreased in all but 1 of the patients at the date of last follow-up.

The underlying pathophysiology of COVID-19 involves a maladaptive immune response to SARS-CoV-2 infection with increased levels of IL-6, IL-10, IL-2 and TNF $\alpha$ produced by macrophages, and fewer $\mathrm{CD} 4^{+}$and $\mathrm{CD} 8^{+} \mathrm{T}$ cells, 
Table 2 Leukocyte counts and inflammatory markers in patients receiving CAP-1002

\begin{tabular}{|c|c|c|c|c|c|c|}
\hline & Patient 1 & Patient 2 & Patient 3 & Patient 4 & Patient 5 & Patient 6 \\
\hline WBC $(\times 1000 / u l)$ & $\begin{array}{l}\text { Day } 0: 12.87 \\
\text { Day 1: } 13.84 \\
\text { Day 2: } 12,99 \\
\text { Day 3: } 12.85 \\
\text { Day 4: } 10.01 \\
\text { Day 5: } 8.08 \\
\text { Day 6: } 5.64 \\
\text { Day } 7: 6.79\end{array}$ & $\begin{array}{l}\text { Day } 0: 5.8 \\
\text { Day 1: } 8.4 \\
\text { Day 2: } 6.39 \\
\text { Day 3: } 9.86 \\
\text { Day 5: } 8.02 \\
\text { Day } 7: 5.63\end{array}$ & $\begin{array}{l}\text { Day 0: } 10.17 \\
\text { Day 1: } 10.35 \\
\text { Day 2: } 8.63 \\
\text { Day 3: } 9.34 \\
\text { Day 6: } 7.50 \\
\text { Day 9: } 4.34\end{array}$ & $\begin{array}{l}\text { Day 0: } 13.79 \\
\text { Day 1: } 13.50 \\
\text { Day 2: } 11.76 \\
\text { Day 4: } 13.07 \\
\text { Day 5: } 11.59 \\
\text { Day 6: } 14.51 \text { (pre 2nd } \\
\text { dose) } \\
\text { Day 7: } 15.67 \text { (post 2nd } \\
\text { dose) } \\
\text { Day 8: } 14.67 \\
\text { Day 18: } 23.13\end{array}$ & $\begin{array}{l}\text { Day } 0: 6.38 \\
\text { Day } 1: 7.24 \\
\text { Day 2: } 9.79 \\
\text { Day 3 } 10.45 \\
\text { Day 4: } 9.75 \\
\text { Day 5: } 8.15 \\
\text { Day 6: } 7.2 \\
\text { Day 10: } 4.69\end{array}$ & $\begin{array}{l}\text { Day } 0: 4.41 \\
\text { Day } 1: 8.32 \\
\text { Day } 2: 7.84 \\
\text { Day 3: } 9.03 \\
\text { Day 4: } 10.78 \\
\text { Day } 5: 13.69\end{array}$ \\
\hline $\begin{array}{l}\text { Lymphocytes }(\times 1000 / \\
\text { ul) }\end{array}$ & $\begin{array}{l}\text { Day 0: } 0.26 \\
\text { Day 1: } 0.55 \\
\text { Day 2: } 0.65 \\
\text { Day 3: } 0.39 \\
\text { Day 5: } 0.73 \\
\text { Day 6: } 1.02\end{array}$ & $\begin{array}{l}\text { Day } 0: 1.40 \\
\text { Day } 1: 1.1 \\
\text { Day 2: } 1.29 \\
\text { Day } 31.09\end{array}$ & $\begin{array}{l}\text { Day 0: } 0.31 \\
\text { Day 1: } 0.41 \\
\text { Day 2: } 0.86 \\
\text { Day 3: } 0.37 \\
\text { Day 6: } 0.55 \\
\text { Day 9: } 0.53\end{array}$ & $\begin{array}{l}\text { Day 0: } 0.82 \\
\text { Day 1: } 1.35 \\
\text { Day 2: } 0.59 \\
\text { Day 3: } 0.52 \\
\text { Day 4: } 0.46 \\
\text { Day 5: } 0.67 \\
\text { Day 6: } 0.43 \text { (pre 2nd } \\
\text { dose) } \\
\text { Day 7: } 0.63 \text { (post 2nd } \\
\text { dose) } \\
\text { Day 8: } 0.59 \\
\text { Day 18: } 0.23\end{array}$ & $\begin{array}{l}\text { Day 0: } 0.33 \\
\text { Day 1: } 0.46 \\
\text { Day 2: } 0.85 \\
\text { Day 3: } 0.91 \\
\text { Day 4: } 0.80 \\
\text { Day 5: } 0.85 \\
\text { Day 6: } 0.79 \\
\text { Day 10: } 0.66\end{array}$ & $\begin{array}{l}\text { Day 0: } 0.54 \\
\text { Day 1: } 0.49 \\
\text { Day 2: } 0.48 \\
\text { Day 3: } 0.45 \\
\text { Day 4: } 0.48 \\
\text { Day 5: } 0.49\end{array}$ \\
\hline $\begin{array}{l}\text { C-reactive protein } \\
(\mathrm{mg} / \mathrm{l})\end{array}$ & $\begin{array}{l}\text { Day } 0: 31.9 \\
\text { Day 1: } 18.8 \\
\text { Day 3: } 19.9 \\
\text { Day 7: } 32.3 \text { (pre 2nd } \\
\text { dose) } \\
\text { Day 9: } 16.0 \text { (post 2nd } \\
\text { dose) }\end{array}$ & $\begin{array}{l}\text { Day } 0: 15.2 \\
\text { Day } 2: 6.1\end{array}$ & $\begin{array}{l}\text { Day 0: } 19.4 \\
\text { Day 1: } 15.2 \\
\text { Day 2: } 18.2 \\
\text { Day 3: } 15.5 \\
\text { Day 6: } 17.0 \\
\text { Day 9: } 8.5\end{array}$ & $\begin{array}{l}\text { Day } 0: 2.0 \\
\text { Day 1: } 2.4 \\
\text { Day 2: } 3.2 \\
\text { Day 3: } 6.9 \\
\text { Day 4: } 4.0 \\
\text { Day 5: } 0.5 \\
\text { Day 6: } 0.3 \text { (pre 2nd } \\
\text { dose) } \\
\text { Day 7: } 0.2 \text { (post 2nd } \\
\text { dose) } \\
\text { Day 8: } 0.3 \\
\text { Day 18: } 54.6\end{array}$ & $\begin{array}{l}\text { Day } 0: 54.6 \\
\text { Day } 1: 27.5 \\
\text { Day } 3: 7.4 \\
\text { Day } 5: 3.6 \\
\text { Day 6: } 2.6 \\
\text { Day 10: } 1.0\end{array}$ & $\begin{array}{l}\text { Day } 0: 80.3 \\
\text { Day 1: } 14.2 \\
\text { Day 2: } 8.6 \\
\text { Day 3: } 13.3 \\
\text { Day 4: } 17.9 \\
\text { Day 5: } 44.6\end{array}$ \\
\hline Ferritin (ng/ml) & $\begin{array}{l}\text { Day 0: } 774.52 \\
\text { Day 1: } 755 \\
\text { Day 2: } 586.26 \\
\text { Day 7: } 522.76 \text { (pre 2nd } \\
\quad \text { dose) } \\
\text { Day 9: } 389.45 \text { (post } \\
\text { 2nd dose) }\end{array}$ & $\begin{array}{l}\text { Day 0: } 2991.52 \\
\text { Day 2: } 1457.84\end{array}$ & $\begin{array}{l}\text { Day 0: } 605.43 \\
\text { Day 1: } 584.26 \\
\text { Day 6: } 695.76 \\
\text { Day 9: } 1029.90\end{array}$ & $\begin{array}{l}\text { Day 0: } 866.81 \\
\text { Day 1: } 615.84 \\
\text { Day 2: } 445.95 \\
\text { Day 4: } 432.93 \\
\text { Day 5: } 455.5 \\
\text { Day 6: } 466.05 \text { (pre 2nd } \\
\text { dose) } \\
\text { Day 7: } 408.5 \text { (post 2nd } \\
\text { dose) } \\
\text { Day 8: } 344.7 \\
\text { Day 11: } 252.89\end{array}$ & $\begin{array}{l}\text { Day } 0: 1281.97 \\
\text { Day 1: } 1090.98 \\
\text { Day 2: } 1,189.54 \\
\text { Day 3: } 1491.9 \\
\text { Day 4: } 2266.0 \\
\text { Day 5: } 1785.3 \\
\text { Day 6: } 1349.8 \\
\text { Day 10: } 912.55\end{array}$ & $\begin{array}{l}\text { Day } 0: 1096.83 \\
\text { Day 1: } 849.10 \\
\text { Day 2: } 798.58 \\
\text { Day 3: } 679.62 \\
\text { Day 4: } 634.50 \\
\text { Day 5: } 688.51\end{array}$ \\
\hline IL-6 (pg/ml) & $\begin{array}{l}\text { Day } 0: 216.7 \\
\text { Day 1: } 124.3 \\
\text { Day 2: } 44.2 \\
\text { Day } 7: 4.0 \text { (pre 2nd } \\
\text { dose) } \\
\text { Day } 9:<3.2 \text { (post 2nd } \\
\text { dose) }\end{array}$ & $\begin{array}{l}\text { Day } 0: 79.8 \\
\text { Day } 1: 90.7 \\
\text { Day } 2: 267.2\end{array}$ & $\begin{array}{l}\text { Day } 0: 421.5 \\
\text { Day 1: } 678.6 \\
\text { Day 6: } 52.7 \\
\text { Day 9: } 7.9\end{array}$ & $\begin{array}{l}\text { Day 0: } 348.3 \\
\text { Day 1: } 482.3 \\
\text { Day 3: } 185.2 \\
\text { Day 5: } 14.9 \\
\text { Day 6: } 7.1 \text { (pre 2nd } \\
\text { dose) } \\
\text { Day 7: } 8 \text { (post 2nd } \\
\text { dose) } \\
\text { Day 11: }<3.2\end{array}$ & $\begin{array}{l}\text { Day } 0: 1,006.9 \\
\text { Day } 1: 588.7 \\
\text { Day } 3: 75.7 \\
\text { Day } 5: 102.4 \\
\text { Day } 10: 77.7\end{array}$ & $\begin{array}{l}\text { Day } 0: 594.7 \\
\text { Day } 1: 900.5 \\
\text { Day } 3: 2573.9 \\
\text { Day } 5: 2381.4\end{array}$ \\
\hline $\mathrm{IL}-1 \alpha(\mathrm{pg} / \mathrm{ml})$ & $\begin{array}{l}\text { Day } 0:<3.9 \\
\text { Day } 1:<3.9 \\
\text { Day } 2:<3.9\end{array}$ & $\begin{array}{l}\text { Day } 0:<3.9 \\
\text { Day } 2:<3.9\end{array}$ & $\begin{array}{l}\text { Day 0:- } \\
\text { Day 1:- } \\
\text { Day 6:- }\end{array}$ & $\begin{array}{l}\text { Day } 0:<3.9 \\
\text { Day } 1:<3.9 \\
\text { Day } 3:<3.9 \\
\text { Day } 5:<3.9\end{array}$ & $\begin{array}{l}\text { Day } 0:<3.9 \\
\text { Day } 1:<3.9 \\
\text { Day } 3:<3.9\end{array}$ & $\begin{array}{l}\text { Day 0:- } \\
\text { Day 1:- } \\
\text { Day 3:- } \\
\text { Day 5:- }\end{array}$ \\
\hline
\end{tabular}


Table 2 (continued)

\begin{tabular}{|c|c|c|c|c|c|c|}
\hline & Patient 1 & Patient 2 & Patient 3 & Patient 4 & Patient 5 & Patient 6 \\
\hline IL-1 $\beta(\mathrm{pg} / \mathrm{ml})$ & $\begin{array}{l}\text { Day } 0:<3.2 \\
\text { Day 1: } 4.5 \\
\text { Day 2: }<3.2 \\
\text { Day 7: -NA } \\
\text { Day 9: -NA }\end{array}$ & $\begin{array}{l}\text { Day } 0:<3.2 \\
\text { Day } 1:<3.2 \\
\text { Day } 2:<3.2\end{array}$ & $\begin{array}{l}\text { Day } 0:<3.2 \\
\text { Day } 1:<3.2 \\
\text { Day } 6:<3.2\end{array}$ & $\begin{array}{l}\text { Day } 0:<3.2 \\
\text { Day } 1:<3.2 \\
\text { Day } 3:<3.2 \\
\text { Day } 5:<3.2\end{array}$ & $\begin{array}{l}\text { Day } 0:<3.2 \\
\text { Day } 1:<3.2 \\
\text { Day } 3:<3.2 \\
\text { Day } 5:<3.2\end{array}$ & $\begin{array}{l}\text { Day } 0:<3.2 \\
\text { Day } 1:<3.2 \\
\text { Day } 3:<3.2 \\
\text { Day } 5:<3.2\end{array}$ \\
\hline $\mathrm{TNF}-\alpha(\mathrm{pg} / \mathrm{ml})$ & $\begin{array}{l}\text { Day 0: } 7.0 \\
\text { Day 1: } 4.5 \\
\text { Day 2: } 13.1\end{array}$ & $\begin{array}{l}\text { Day } 0: 14.6 \\
\text { Day } 1:- \\
\text { Day } 2: 30.1\end{array}$ & $\begin{array}{l}\text { Day } 0: 46.7 \\
\text { Day 1: } 30.5 \\
\text { Day 6: } 24.3\end{array}$ & $\begin{array}{l}\text { Day 0: } 52.5 \\
\text { Day 1: } 55.6 \\
\text { Day 3: } 35.9 \\
\text { Day 5: } 13.6 \\
\text { Day 7: } 29.2 \text { (post 2nd } \\
\text { dose) } \\
\text { Day 11: } 19.6\end{array}$ & $\begin{array}{l}\text { Day } 0: 15.6 \\
\text { Day 1: } 20.4 \\
\text { Day 3: } 22.8 \\
\text { Day 5: } 25.2 \\
\text { Day 10: } 16.0\end{array}$ & $\begin{array}{l}\text { Day 0: } 17.9 \\
\text { Day 1: }- \\
\text { Day 3: } 49.4 \\
\text { Day 5: } 22.9\end{array}$ \\
\hline IL-10 (pg/ml) & $\begin{array}{l}\text { Day } 0:<3.2 \\
\text { Day } 1:<3.2 \\
\text { Day } 2:<3.2 \\
\text { Day } 7:<3.2 \text { (pre } 2 \text { nd } \\
\text { dose) } \\
\text { Day } 9:<3.2 \text { (post } 2 \text { nd } \\
\text { dose) }\end{array}$ & $\begin{array}{l}\text { Day } 0: 6.1 \\
\text { Day } 1: 3.5 \\
\text { Day } 2: 10.3\end{array}$ & $\begin{array}{l}\text { Day } 0: 7.5 \\
\text { Day } 1: 4.8 \\
\text { Day } 6:<3.2 \\
\text { Day } 9:<3.2\end{array}$ & $\begin{array}{l}\text { Day } 0: 6.3 \\
\text { Day 1: } 7.0 \\
\text { Day } 3: 3.3 \\
\text { Day 5: }<3.2 \\
\text { Day } 7: 10.2 \text { (post 2nd } \\
\quad \text { dose) } \\
\text { Day } 11:<3.2\end{array}$ & $\begin{array}{l}\text { Day } 0: 8.6 \\
\text { Day 1: } 14.8 \\
\text { Day 3: } 9.5 \\
\text { Day 5: } 8\end{array}$ & $\begin{array}{l}\text { Day 0: } 11.6 \\
\text { Day 1: } 15.1 \\
\text { Day 3: } 16.7 \\
\text { Day 5: } 14.8\end{array}$ \\
\hline
\end{tabular}

Day 0 shows laboratory values prior to the first administration of CAP-1002. Patients 1 and 4 received a second dose of CAP-1002, 7 days after the first infusion
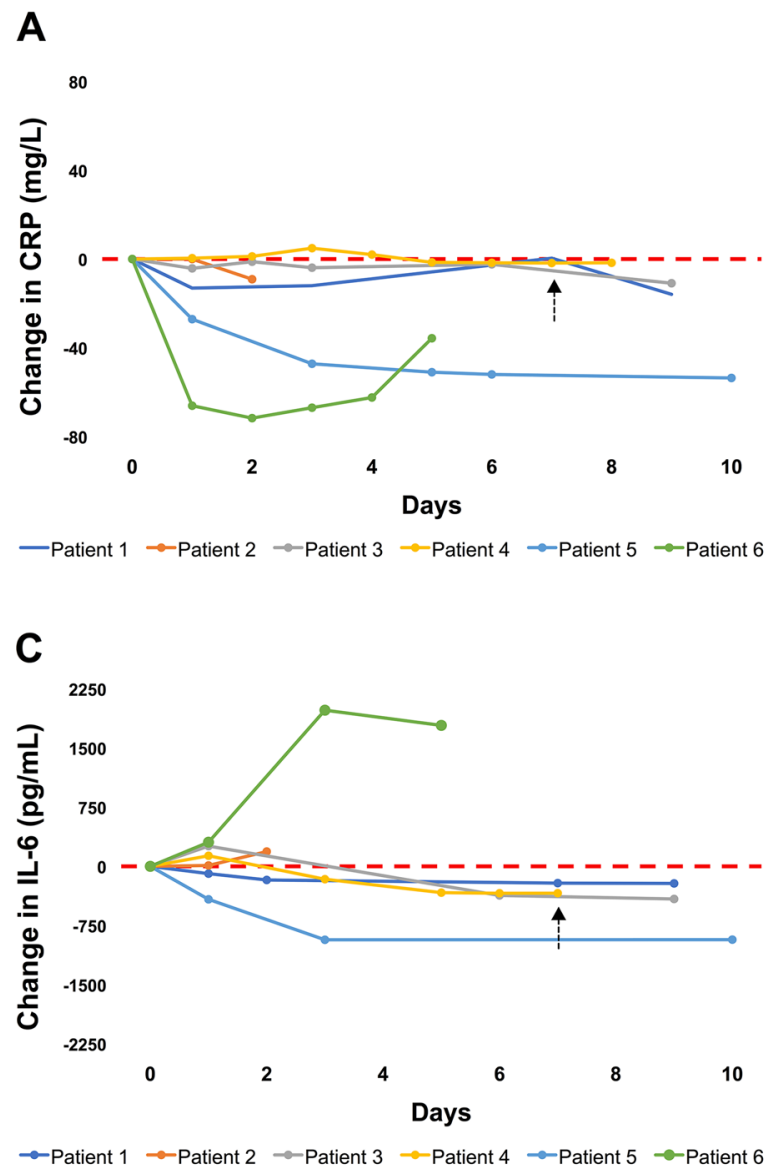

Fig. 2 Trends in inflammatory markers and lymphocyte counts. Changes in the level of inflammatory markers, including C-reactive protein (a), ferritin (b), IL-6 (c), and lymphocyte counts (d) within
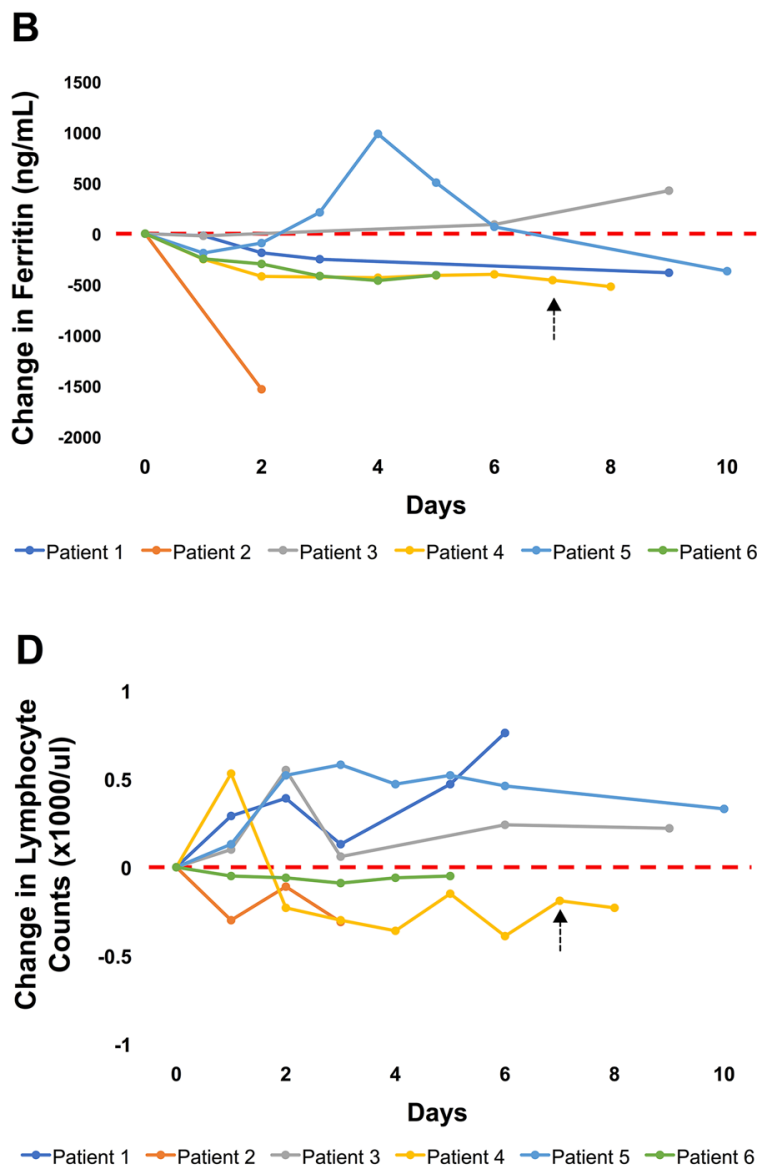

10 days of CAP-1002 infusion, normalized to baseline values (preinfusion). Dashed arrows indicated the time of second infusion 
Fig. 3 Clinical course of patients receiving CAP-1002. The clinical course is plotted for individual patients on the 7-point WHO scale for evaluation of patients with COVID-19. Day 0 denotes time of CAP1002 infusion

\begin{tabular}{|l|c|l|l|l|l|l|l|}
\hline & $\begin{array}{c}\text { WHO } \\
\text { Class }\end{array}$ & Patient \# 1 & Patient \# 2 & Patient \# 3 & Patient \# 4 & Patient \# 5 & Patient \# 6 \\
\hline Death & 7 & & & & & & \\
\hline $\begin{array}{l}\text { Hospitalized, requiring ECMO, invasive } \\
\text { mechanical ventilation, or both }\end{array}$ & 6 & & Day 0-3 & Day 0-3 & $\begin{array}{l}\text { Ongoing: } \\
\text { Day 0-18 }\end{array}$ & Day 0 & \\
\hline $\begin{array}{l}\text { Hospitalized, requiring nasal high-flow oxygen } \\
\text { therapy, noninvasive mechanical ventilation, or } \\
\text { both }\end{array}$ & 5 & Day 0-7 & & & & Day 1 & $\begin{array}{l}\text { Ongoing: } \\
\text { Day 0-5 }\end{array}$ \\
\hline Hospitalized, requiring supplemental oxygen & 4 & Day 8 & Day 4 & Day 4 & & Day 3 & \\
\hline Hospitalized, not requiring supplemental oxygen & 3 & Day 11 & Day 7 & Day 11 & & Day 4 & \\
\hline Discharged, unable to resume normal activities & 2 & Day 11 & Day 9 & Day 17 & & Day 11 & \\
\hline Discharged, resumption of normal activities & 1 & Day 15 & Day 13 & & & & \\
\hline
\end{tabular}

Table 3 Outcomes and characteristics of CAP-1002-treated COVID-19 patients and a contemporaneous control group

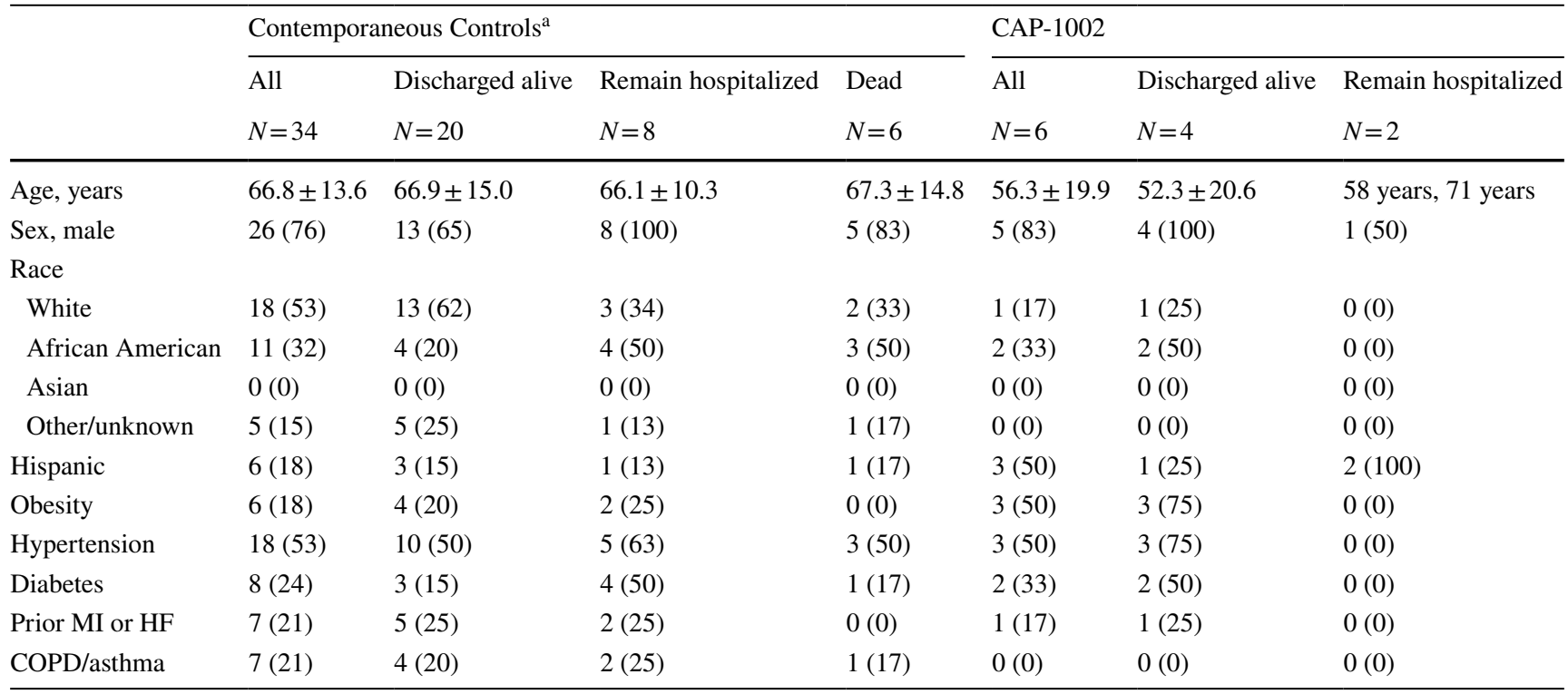

COPD chronic obstructive pulmonary disease, COVID-19 coronavirus disease 2019, $H F$ heart failure, $M I$ myocardial infarction

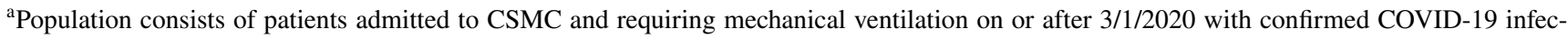
tion. Patients were excluded if they: (1) did not have at least 30.7 days of follow-up from admission to the terminal event (death or hospital discharge), in order to match the follow-up duration in the CAP-1002 group; (2) were enrolled in a clinical trial requiring informed consent; (3) did not receive an IL-6 inhibitor; or (4) had a tracheostomy placed prior to the current admission. Due to small sample sizes, statistical tests for comparison were not performed. Categorical data presented as total count and percentage (\%), and continuous data are presented as mean \pm standard deviation (SD)

but no significant changes in B-cell counts $[1,9,43]$. The dysregulated immune function with cytokine storm leads to lung, heart, and other end-organ injury [22]. Extensive preclinical and some clinical studies suggest that cell therapy may attenuate inflammation [30].

CDCs are stromal progenitor cells isolated from human heart tissue through well-specified culture techniques and exert their effects in a paracrine manner by secreting exosomes (nanosized vesicles with bioactive payload) [16, 17, 31, 39]. CDCs target multiple cytokine pathways (e.g., TNF $\alpha$, IFN- $\gamma$, IL-1 $\beta$, IL-6) that are associated with disease progression and poor outcomes in COVID-19 (Fig. 1). For example, CDCs have the capacity to polarize macrophages toward an anti-inflammatory and healing phenotype [30]. 
These anti-inflammatory effects have been demonstrated in animal models of myocardial ischemia, myocarditis, muscular dystrophy, aging, heart failure with preserved ejection fraction, pulmonary arterial hypertension and dilated cardiomyopathy [3, 20, 21, 33, 34, 42]. Finally, based on preclinical work, a majority of IV CDCs are retained in the lungs [6]. Thus, there may be local benefits within the lung parenchyma, although this remains a conjecture.

A number of as-yet-unaddressed issues pertinent to use of cell therapy in SARS-CoV-2 infection need to be highlighted. These include the optimal sources of cells and dosing strategies. With respect to safety, no serious adverse events have been noted with administration of CAP-1002 or MSCs from various sources in patients. Another issue related to safety is the potential secretion of IL- 6 by CDCs [32]. This potential source of IL-6, however, appears to be negligible, as multiple preclinical models demonstrated the opposite effect following CDC administration: significantly decreased serum levels of IL-6 after CDC infusion [21, 24]. Thus, future studies should logically focus on recognized endpoints including overall mortality, survival to hospital discharge and beyond, length of hospital stay and ventilator-free days, with proper randomization and rigorous controls. A composite ordinal scale reflecting improvement in COVID-19 disease state may trenchantly capture the key clinical outcomes (as recommended by the WHO R\&D Blueprint) [46].

\section{Limitations}

Our study has several limitations. The absence of a true, randomized control group and the small sample size prevent any meaningful inferences about cause-effect relationship between receipt of CAP-1002 and clinical improvement. As per the standard care protocols for critically ill COVID-19 patients at CSMC, the patients enrolled in the study were receiving concomitant anti-viral and anti-inflammatory therapies, which may have confounded the positive clinical outcomes and introduced heterogeneity into assessments of circulating biomarkers. Nevertheless, we are encouraged by the higher survival rates in the CAP-1002 group when compared against a contemporaneous cohort that similarly received anti-IL-6 therapy and hydroxychloroquine. Furthermore, two drugs that were administered in our cohort of 6 patients and could have a potential confounding effect (lopinavir/ritonavir and hydroxychloroquine) were recently demonstrated to have no efficacy in COVID-19 patients [8, 28]. Remdesivir was not used in any of the patients reported here. These observations, however, do not definitively eliminate potential confounding.

Despite these limitations, the encouraging clinical outcomes and biochemical responses in the COVID19 patients treated with CAP-1002 warrant further investigation in prospective registries and randomized placebo-controlled clinical trials. As an immediate successor to the present compassionate-use study, $\mathbf{C d c S}$ for Cytokine Storm in Covid Syndrome Trial: $(\mathbf{C S})^{\mathbf{3}}$ is an expanded access protocol that is now exploring clinical and biochemical effects of CAP-1002 administration in patients with severe COVID-19 infection, under an Investigational New Drug application awarded April 22, 2020 [14]. A randomized controlled trial evaluating CAP-1002 versus placebo for the treatment of severe COVID-19 is currently in the advanced planning stages.

\section{Conclusions}

In this initial series of six patients with severe COVID-19 and associated lung injury, administration of CAP-1002 was safely tolerated, and followed by improvement in clinical status and reductions in selective pro-inflammatory biomarkers in most patients. The small sample size and lack of a true, randomized control arm prevent conclusive assessment of this treatment modality-larger expanded use assessments and, most importantly, randomized placebocontrolled trials will more definitively assess this therapy.

Acknowledgements We thank Tracey Early for assistance with recruitment and data analysis.

Author contributions RRM, EM, TC, and SS contributed to the design of the study. SS, TC, PC, AA, JF, OF, TZ, JEE, MG, LM, EM, and RRM contributed to data analysis/interpretation and drafting of the manuscript. EM and AA contributed to critical revision of the manuscript.

Funding Expenses specific to the clinical study, and analyses of the clinical data, were funded by a gift from the Smidt Family Foundation. Capricor provided regulatory support for the study and the cell product, CAP-1002, free of charge for this study. EM holds the Mark S. Siegel Family Foundation Distinguished Chair, and RRM holds the Stephen R. Corday, MD Chair in Interventional Cardiology.

Availability of data and material All described data are included within the manuscript. Any additional data are available upon request.

\section{Compliance with ethical standards}

Conflict of interest EM owns founder's equity in Capricor Therapeutics. LM is employed by Capricor and owns equity in the company. Other authors report no conflicts.

Ethics approval The study was approved by the Institutional Review Board at Cedars-Sinai Medical Center.

Informed consent Each patient (or their legal representative) gave written informed consent to participate.

Open Access This article is licensed under a Creative Commons Attribution 4.0 International License, which permits use, sharing, 
adaptation, distribution and reproduction in any medium or format, as long as you give appropriate credit to the original author(s) and the source, provide a link to the Creative Commons licence, and indicate if changes were made. The images or other third party material in this article are included in the article's Creative Commons licence, unless indicated otherwise in a credit line to the material. If material is not included in the article's Creative Commons licence and your intended use is not permitted by statutory regulation or exceeds the permitted use, you will need to obtain permission directly from the copyright holder. To view a copy of this licence, visit http://creativecommons .org/licenses/by/4.0/.

\section{References}

1. Akhmerov A, Marban E (2020) COVID-19 and the heart. Circ Res. https://doi.org/10.1161/circresaha.120.317055

2. Aminzadeh MA, Rogers RG, Fournier M, Tobin RE, Guan X, Childers MK, Andres AM, Taylor DJ, Ibrahim A, Ding X, Torrente A, Goldhaber JM, Lewis M, Gottlieb RA, Victor RA, Marbán E (2018) Exosome-mediated benefits of cell therapy in mouse and human models of duchenne muscular dystrophy. Stem Cell Rep 10:942-955. https://doi.org/10.1016/j.stemc r.2018.01.023

3. Aminzadeh MA, Tseliou E, Sun B, Cheng K, Malliaras K, Makkar RR, Marbán E (2015) Therapeutic efficacy of cardiosphere-derived cells in a transgenic mouse model of non-ischaemic dilated cardiomyopathy. Eur Heart J 36:751-762. https:// doi.org/10.1093/eurheartj/ehu196

4. Definition Task Force ARDS, Ranieri VM, Rubenfeld GD, Thompson BT, Ferguson ND, Caldwell E, Fan E, Camporota L, Slutsky AS (2012) Acute respiratory distress syndrome: the Berlin Definition. JAMA 307:2526-2533. https://doi. org/10.1001/jama.2012.5669

5. Arentz M, Yim E, Klaff L, Lokhandwala S, Riedo FX, Chong M, Lee M (2020) Characteristics and outcomes of 21 critically ill patients with COVID-19 in Washington State. JAMA. https ://doi.org/10.1001/jama.2020.4326

6. Bonios M, Terrovitis J, Chang CY, Engles JM, Higuchi T, Lautamäki R, Yu J, Fox J, Pomper M, Wahl RL, Tsui BM, O’Rourke B, Bengel FM, Marbán E, Abraham MR (2011) Myocardial substrate and route of administration determine acute cardiac retention and lung bio-distribution of cardiosphere-derived cells. J Nucl Cardiol 18:443-450. https://doi.org/10.1007/s1235 0-011-9369-9

7. Bhatraju PK, Ghassemieh BJ, Nichols M, Kim R, Jerome KR, Nalla AK, Greninger AL, Pipavath S, Wurfel MM, Evans L, Kritek PA, West TE, Luks A, Gerbino A, Dale CR, Goldman JD, O’Mahony S, Mikacenic C (2020) Covid-19 in critically ill patients in the Seattle Region-case series. N Engl J Med. https ://doi.org/10.1056/nejmoa2004500

8. Cao B, Wang Y, Wen D, Liu W, Wang J, Fan G, Ruan L, Song B, Cai Y, Wei M, Li X, Xia J, Chen N, Xiang J, Yu T, Bai T, Xie X, Zhang L, Li C, Yuan Y, Chen H, Li H, Huang H, Tu S, Gong F, Liu Y, Wei Y, Dong C, Zhou F, Gu X, Xu J, Liu Z, Zhang Y, Li H, Shang L, Wang K, Li K, Zhou X, Dong X, Qu Z, Lu S, Hu X, Ruan S, Luo S, Wu J, Peng L, Cheng F, Pan L, Zou J, Jia C, Wang J, Liu X, Wang S, Wu X, Ge Q, He J, Zhan H, Qiu F, Guo L, Huang C, Jaki T, Hayden FG, Horby PW, Zhang D, Wang C (2020) A trial of lopinavir-ritonavir in adults hospitalized with severe Covid-19. N Engl J Med. https://doi.org/10.1056/nejmo a2001282

9. Cao X (2020) COVID-19: immunopathology and its implications for therapy. Nat Rev Immunol. https://doi.org/10.1038/ s41577-020-0308-3
10. Centers for Disease Control and Prevention. Evaluating and Testing Persons for Coronavirus Disease 2019 (COVID-19). Available at: https://www.cdc.gov/coronavirus/2019-nCoV/hcp/ clinical-criteria.html. Accessed 28 Apr 2020

11. Chakravarty T, Makkar RR, Ascheim DD, Traverse JH, Schatz R, DeMaria A, Francis GS, Povsic TJ, Smith RR, Lima JA, Pogoda JM, Marbán L, Henry TD (2017) Allogeneic heart stem cells to achieve myocardial regeneration (ALLSTAR) trial: rationale and design. Cell Transplant 26:205-214. https://doi. org/10.3727/096368916X692933

12. Chen J, Hu C, Chen L, Tang L, Zhu Y, Xu X, Chen L, Gao H, Lu X, Yu L, Dai X, Xiang C, Li L (2020) Clinical study of mesenchymal stem cell treatment for acute respiratory distress syndrome induced by epidemic influenza A (H7N9) infection: a hint for COVID-19 treatment. Engineering (Beijing). https:// doi.org/10.1016/j.eng.2020.02.006

13. Cheng K, Ibrahim A, Hensley MT, Shen D, Sun B, Middleton R, Liu W, Smith RR, Marbán E (2014) Relative roles of CD90 and c-kit to the regenerative efficacy of cardiosphere-derived cells in humans and in a mouse model of myocardial infarction. J Am Heart Assoc 3:e001260. https://doi.org/10.1161/JAHA.114.00126 0

14. ClinicalTrials.gov. CAP-1002 in Severe COVID-19 Disease. 2020; NCT04338347. Available at: https://clinicaltrials.gov/ct2/show/ NCT04338347. Accessed 19 Apr 2020

15. de Couto G (2019) Macrophages in cardiac repair: environmental cues and therapeutic strategies. Exp Mol Med 51:1-10. https://doi. org/10.1038/s12276-019-0269-4

16. de Couto G, Gallet R, Cambier L, Jaghatspanyan E, Makkar N, Dawkins JF, Berman BP, Marbán E (2017) Exosomal microRNA transfer into macrophages mediates cellular postconditioning. Circulation 136:200-214. https://doi.org/10.1161/circulatio naha.116.024590

17. de Couto G, Liu W, Tseliou E, Sun B, Makkar N, Kanazawa H, Arditi M, Marbán E (2015) Macrophages mediate cardioprotective cellular postconditioning in acute myocardial infarction. J Clin Invest 125:3147-3162. https://doi.org/10.1172/JCI81321

18. Donoghue M, Hsieh F, Baronas E, Godbout K, Gosselin M, Stagliano N, Donovan M, Woolf B, Robison K, Jeyaseelan R, Breitbart RE, Acton S (2000) A novel angiotensin-converting enzyme-related carboxypeptidase (ACE2) converts angiotensin I to angiotensin 1-9. Circ Res 87:E1-9. https://doi.org/10.1161/01. res.87.5.e1

19. Ferreira FL, Bota DP, Bross A, Melot C, Vincent JL (2001) Serial evaluation of the SOFA score to predict outcome in critically ill patients. JAMA 286:1754-1758. https://doi.org/10.1001/ jama.286.14.1754

20. Gallet R, Dawkins J, Valle J, Simsolo E, de Couto G, Middleton R, Tseliou E, Luthringer D, Kreke M, Smith RR, Marbán L, Ghaleh B, Marbán E (2017) Exosomes secreted by cardiosphere-derived cells reduce scarring, attenuate adverse remodelling, and improve function in acute and chronic porcine myocardial infarction. Eur Heart J 38:201-211. https://doi.org/10.1093/eurheartj/ehw240

21. Gallet R, de Couto G, Simsolo E, Valle J, Sun B, Liu W, Tseliou E, Zile MR, Marbán E (2016) Cardiosphere-derived cells reverse heart failure with preserved ejection fraction $(\mathrm{HFpEF})$ in rats by decreasing fibrosis and inflammation. JACC Basic Transl Sci 1:14-28. https://doi.org/10.1016/j.jacbts.2016.01.003

22. Gori T, Lelieveld J, Münzel T (2020) Perspective: cardiovascular disease and the Covid-19 pandemic. Basic Res Cardiol 115:32. https://doi.org/10.1007/s00395-020-0792-4

23. Grens K. TheScientist (2020) The Lancet Retracts Cardiac Stem Cell Clinical Trial Paper. 2019. Available at: https://www.thescientist.com/news-opinion/the-lancet-retracts-cardiac-stem-cellclinical-trial-paper-65598. Accessed 19 Apr 2020 
24. Grigorian-Shamagian L, Liu W, Fereydooni S, Middleton RC, Valle J, Cho JH, Marbán E (2017) Cardiac and systemic rejuvenation after cardiosphere-derived cell therapy in senescent rats. Eur Heart J 38:2957-2967. https://doi.org/10.1093/eurheartj/ehx454

25. Huang C, Wang Y, Li X, Ren L, Zhao J, Hu Y, Zhang L, Fan G, Xu J, Gu X, Cheng Z, Yu T, Xia J, Wei Y, Wu W, Xie X, Yin W, Li H, Liu M, Xiao Y, Gao H, Guo L, Xie J, Wang G, Jiang R, Gao Z, Jin Q, Wang J (2020) Cao B (2020) Clinical features of patients infected with 2019 novel coronavirus in Wuhan. China Lancet 395:497-506. https://doi.org/10.1016/S0140-6736(20)30183-5

26. Leng Z, Zhu R, Hou W, Feng Y, Yang Y, Han Q, Shan G, Meng F, Du D, Wang S, Fan J, Wang W, Deng L, Shi H, Li H, Hu Z, Zhang F, Gao J, Liu H, Li X, Zhao Y, Yin K, He X, Gao Z, Wang Y, Yang B, Jin R, Stambler I, Lim LW, Su H, Moskalev A, Cano A, Chakrabarti S, Min KJ, Ellison-Hughes G, Caruso C, Jin K, Zhao RC (2020) Transplantation of ACE2(-) mesenchymal stem cells improves the outcome of patients with COVID-19 pneumonia. Aging Dis 11:216-228. https://doi.org/10.14336/AD.2020.0228

27. Li TS, Cheng K, Malliaras K, Smith RR, Zhang Y, Sun B, Matsushita N, Blusztajn A, Terrovitis J, Kusuoka H, Marbán L, Marbán E (2012) Direct comparison of different stem cell types and subpopulations reveals superior paracrine potency and myocardial repair efficacy with cardiosphere-derived cells. J Am Coll Cardiol 59:942-953. https://doi.org/10.1016/j.jacc.2011.11.029

28. Magagnoli J, Narendran S, Pereira F, Cummings T, Hardin JW, Sutton SS, Ambati J (2020) Outcomes of hydroxychloroquine usage in United States veterans hospitalized with Covid-19. medRxiv. https://doi.org/10.1101/2020.04.16.20065920

29. Makkar RR, Smith RR, Cheng K, Malliaras K, Thomson LE, Berman D, Czer LS, Marbán L, Mendizabal A, Johnston PV, Russell SD, Schuleri KH, Lardo AC, Gerstenblith G, Marbán E (2012) Intracoronary cardiosphere-derived cells for heart regeneration after myocardial infarction (CADUCEUS): a prospective, randomised phase 1 trial. Lancet 379:895-904. https://doi. org/10.1016/S0140-6736(12)60195-0

30. Marbán E (2018) A mechanistic roadmap for the clinical application of cardiac cell therapies. Nat Biomed Eng 2:353-361. https ://doi.org/10.1038/s41551-018-0216-Z

31. Marbán E (2018) The secret life of exosomes: what bees can teach us about next-generation therapeutics. J Am Coll Cardiol 71:193-200. https://doi.org/10.1016/j.jacc.2017.11.013

32. Maxeiner H, Mufti S, Krehbiehl N, Dülfer F, Helmig S, Schneider J, Böning A, Matejec R, Weigand MA, Schlüter KD, Wenzel S (2014) Interleukin-6 contributes to the paracrine effects of cardiospheres cultured from human, murine and rat hearts. J Cell Physiol 229:1681-1689. https://doi.org/10.1002/jcp.24613

33. Middleton RC, Fournier M, Xu X, Marban E, Lewis MI (2017) Therapeutic benefits of intravenous cardiosphere-derived cell therapy in rats with pulmonary hypertension. PLoS ONE 12(8):e0183557. https://doi.org/10.1371/journal.pone.0183557

34. Nana-Leventaki E, Nana M, Poulianitis N, Sampaziotis D, Perrea D, Sanoudou D, Rontogianni D, Malliaras K (2019) Cardiospherederived cells attenuate inflammation, preserve systolic function, and prevent adverse remodeling in rat hearts with experimental autoimmune myocarditis. J Cardiovasc Pharmacol Ther 24:70-77. https://doi.org/10.1177/1074248418784287

35. Qin C, Zhou L, Hu Z, Zhang S, Yang S, Tao Y, Xie C, Ma K, Shang K, Wang W, Tian DS (2020) Dysregulation of immune response in patients with COVID-19 in Wuhan, China. Clin Infect Dis. https://doi.org/10.1093/cid/ciaa248

36. Reich H, Tseliou E, de Couto G, Angert D, Valle J, Kubota Y, Luthringer D, Mirocha J, Sun B, Smith RR, Marbán L, Marbán E (2016) Repeated transplantation of allogeneic cardiospherederived cells boosts therapeutic benefits without immune sensitization in a rat model of myocardial infarction. J Heart Lung Transplant 35:1348-1357. https://doi.org/10.1016/j.healun.2016.05.008
37. Richardson S, Hirsch JS, Narasimhan M, Crawford JM, McGinn T, Davidson KW, and the Northwell COVID-19 Research Consortium, Barnaby DP, Becker LB, Chelico JD, Cohen SL, Cookingham J, Coppa K, Diefenbach MA, Dominello AJ, Duer-Hefele J, Falzon L, Gitlin J, Hajizadeh N, Harvin TG, Hirschwerk DA, Kim EJ, Kozel ZM, Marrast LM, Mogavero JN, Osorio GA, Qiu M, Zanos TP (2020) Presenting characteristics, comorbidities, and outcomes among 5700 patients hospitalized with COVID19 in the New York City area. JAMA. https://doi.org/10.1001/ jama.2020.6775

38. Rizzo P, Vieceli Dalla Sega F, Fortini F, Marracino L, Rapezzi C, Ferrari R (2020) COVID-19 in the heart and the lungs: could we "Notch" the inflammatory storm? Basic Res Cardiol 115:31. https://doi.org/10.1007/s00395-020-0791-5

39. Rogers RG, Fournier M, Sanchez L, Ibrahim AG, Aminzadeh MA, Lewis MI, Marbán E (2019) Disease-modifying bioactivity of intravenous cardiosphere-derived cells and exosomes in $\mathrm{mdx}$ mice. JCI Insight. https://doi.org/10.1172/jci.insight.130202

40. Ruan Q, Yang K, Wang W, Jiang L, Song J (2020) Clinical predictors of mortality due to COVID-19 based on an analysis of data of 150 patients from Wuhan, China. Intensive Care Med. https:// doi.org/10.1007/s00134-020-06028-z

41. Smith RR, Barile L, Cho HC, Leppo MK, Hare JM, Messina E, Giacomello A, Abraham MR, Marbán E (2007) Regenerative potential of cardiosphere-derived cells expanded from percutaneous endomyocardial biopsy specimens. Circulation 115:896-908. https://doi.org/10.1161/CIRCULATIONAHA.106.655209

42. Taylor M, Jefferies J, Byrne B, Lima J, Ambale-Venkatesh B, Ostovaneh MR, Makkar R, Goldstein B, Smith RR, Fudge J, Malliaras K, Fedor B, Rudy J, Pogoda JM, Marbán L, Ascheim DD, Marbán E, Victor RG (2019) Cardiac and skeletal muscle effects in the randomized HOPE-Duchenne trial. Neurology 92:e866e878. https://doi.org/10.1212/WNL.0000000000006950

43. Vaninov $N$ (2020) In the eye of the COVID-19 cytokine storm. Nat Rev Immunol. https://doi.org/10.1038/s41577-020-0305-6

44. Wang D, Hu B, Hu C, Zhu F, Liu X, Zhang J, Wang B, Xiang H, Cheng Z, Xiong Y, Zhao Y, Li Y, Wang X, Peng Z (2020) Clinical characteristics of 138 hospitalized patients with 2019 novel Coronavirus-infected pneumonia in Wuhan, China. JAMA. https ://doi.org/10.1001/jama.2020.1585

45. WHO (2020) Coronavirus disease (COVID-10) Pandemic. Available at: https://www.who.int/emergencies/diseases/novel-coron avirus-2019. Accessed 28 Apr 2020

46. WHO (2019) WHO R\&D Blueprint. COVID-19 Therapeutic Trial Synopsis. 2020. Available at: https://www.who.int/blueprint/ priority-diseases/key-action/COVID-19_Treatment_Trial_Desig n_Master_Protocol_synopsis_Final_18022020.pdf. Accessed 19 Apr 2020

47. Wu Z, McGoogan JM (2020) Characteristics of and important lessons from the coronavirus disease 2019 (COVID-19) outbreak in China: summary of a report of 72314 cases from the Chinese Center for Disease Control and Prevention. JAMA. https://doi. org/10.1001/jama.2020.2648

48. Yang X, Yu Y, Xu J, Shu H, Xia J, Liu H, Wu Y, Zhang L, Yu Z, Fang M, Yu T, Wang Y, Pan S, Zou X, Yuan S, Shang Y (2020) Clinical course and outcomes of critically ill patients with SARS-CoV-2 pneumonia in Wuhan, China: a single-centered, retrospective, observational study. Lancet Respir Med. https://doi. org/10.1016/S2213-2600(20)30079-5

49. Young BE, Ong SWX, Kalimuddin S, Low JG, Tan SY, Loh J, Ng OT, Marimuthu K, Ang LW, Mak TM, Lau SK, Anderson DE, Chan KS, Tan TY, Ng TY, Cui L, Said Z, Kurupatham L, Chen MI, Chan M, Vasoo S, Wang LF, Tan BH, Lin RTP, Lee VJM, Leo YS, Lye DC (2020) Epidemiologic features and clinical course of patients infected with SARS-CoV-2 in Singapore. JAMA. https:// doi.org/10.1001/jama.2020.3204 